\title{
Dynamic Motion Planning Whole Arm Grasp Systems Based on Switching Contact Modes
}

\author{
Masahito Yashima, Hideya Yamaguchi \\ National Defense Academy, Yokosuka 239-8686 JAPAN \\ \{ yashima, hideya $\} @$ nda.ac.jp
}

\begin{abstract}
We present motion planning for dexterous manipulation by whole arm grasp system based on switching contact modes. Motion planning for such a system should consider changing dynamics and kinematics according to the contact modes. We systematize the properties of the manipulation system by taking account of contact dynamics and kinematics, and derive the conditions to be satisfied in motion planning. The conditions give the restrictions of the feasible contact mode and the number of contact points. Noting that the manipulation system which involves continuous $d y$ namics and discrete change of contact modes belongs to a class of hybrid system, we propose an algorithm of global motion planning based on a hybrid control perspective. We aim at finding a feasible joint torque trajectory and a sequence of contact modes to move the object to an object final state for given switching times and object state trajectory. The proposed planning algorithm mainly consists of planning the object trajectory by a randomized approach using an object nominal trajectory, and constructing subgoals which satisfy the proposed conditions by solving an inverse problem. We show the validity of the planner by simulating manipulation by whole arm grasp system.
\end{abstract}

\section{Introduction}

Manipulating objects by hands, we dexterously use different contact modes such as rolling and sliding contacts unconsciously. Utilizing different contact modes according to the manipulation task can raise the manipulation skill with the hands. The primary motivation of our work is to manipulate objects by a multi-fingered robot hand utilizing different contact modes. In this paper, we discuss motion planning to realize such a manipulation as mentioned above.

Most work on motion planning has been devoted to the problem of deciding a collision-free path that connects a given start and goal configuration for movable objects or manipulators by searching a configuration space while satisfying geometrical constraints imposed by obstacles[1],[2],[7]. This work does not deal with any dynamics for robots or objects. In other words, the conventional motion planning is based on a geometrical approach.
Recently there have been some work on motion planning that determines control inputs to derive a robot from an initial state to a goal state while avoiding obstacles and obeying dynamics of robots[8],[9]. There is less work on planning for the motion occurring contacts between objects. Such motion planning is a complicated problem because of the nature of the contact such as rolling and sliding.

Work on manipulation by a multi-fingered robot hand has attracted attention. Most work has been devoted to the instantaneous kinematic and dynamic analysis of grasping and dexterous manipulation, assuming that there is no change of contact modes between hand and objects during a task. In contrast to the conventional manipulation, we should take account of changing dynamics and kinematics of the manipulation system according to the change of contact modes and verify the dynamical and kinematical properties of such a manipulation system in order to achieve our goal. Trinkle and Hunter[13] showed global motion planning for quasi-static manipulation by searching feasible contact configurations such as edge-edge, edge-vertex contacts and so on. However, since contacts are restricted to frictionless contacts, the planner can not be applied to motion planning of manipulation with changing contact modes. Cherif and Gupta[4] presented global motion planning for quasi-static fingertip manipulation with changing contact modes and discussed a planning algorithm based on the idea of an inverse finger motion problem. The idea motivated us to consider the inverse problem for the motion planning problem of whole arm grasp system. However the use of their algorithm is limited because they discussed the transition of contact modes without considering contact dynamics. A crucial issue for motion planing of the manipulation system with switching contact modes is to find the feasible trajectories (motion and joint driving torques) which satisfy the dynamical and kinematical constraints at contact points between the hands and object. In addition, designing an efficient algorithm for exploring high dimensional search space is required since we should deal with the state space of the manipulation system.

For a system with changing dynamics behavior, the state space of manipulation system consists of multiple partitions, each of which corresponds to a contact mode. Each 
partition is governed by a different set of differential equations. In order to complete successful manipulation, it is necessary to plan transitions of the contact modes. Since the manipulation system can be modeled by a discrete event such as a contact mode and continuous dynamics, the system belongs to a class of hybrid system[3],[10]. Most work on the hybrid system is the stability and modeling from the view of control theory. Zefran[15] applied the idea of hybrid system to the study of grasp gaits. To my knowledge, there is no study about motion planning for dexterous manipulation with switching contact modes from a hybrid control perspective.

In this paper, we first develop mathematical conditions to be satisfied for motion planning, based on dynamics and kinematics of manipulation system. These conditions give restrictions for feasible contact modes and the number of contact points. We then describe the basic ideas of manipulation planning, which is defined as "given initial and goal object states, it is to find a joint torque trajectory, using rolling and sliding contacts". The proposed algorithm is simplified using a randomized technique. Finally we apply the ideas to the simulation of a whole arm grasp system.

\section{Problem formulation}

\subsection{Dynamic model}

We derive the dynamic model of whole arm grasp system in space as shown in Figure 1. We assume that each link of each hand (arm) has one contact point with an object at most, and each revolute joint has one degree of freedom. Suppose there are $n_{C}$ contacts, consisting of $n_{R}$ rolling contacts and $n_{S}$ sliding contacts. Let the subscripts $N$, $T$ and $U$ denote the inward normal and two tangential directions spanning tangent plane at the contact point on the object's surface, and $S$ and $R$ denote sliding and rolling contacts, respectively. A contact frame is assigned to each contact point and is positioned with its origin at the contact point and with its axes aligned with $N, T$ and $U$, respectively. We denote $i$ th contact point by subscript $i$.

The dynamic equation of motion of rigid object can be written as

$$
\boldsymbol{M}_{o} \ddot{\boldsymbol{q}}=\boldsymbol{G}_{N} \boldsymbol{f}_{N}+\boldsymbol{G}_{T} \boldsymbol{f}_{T}+\boldsymbol{G}_{U} \boldsymbol{f}_{U}+\boldsymbol{g}_{o}
$$

where $\ddot{q}$ is the 6-vector of linear and angular acceleration of the center of mass of the object, $\boldsymbol{M}_{o}$ is the $6 \times 6$-mass matrix of the object, $\boldsymbol{g}_{o}$ is external wrench applied to the object, and $\boldsymbol{f}_{N}, \boldsymbol{f}_{T}$ and $\boldsymbol{f}_{U}$ are the normal and tangential contact force vectors expressed in the corresponding contact frame. $\boldsymbol{G}_{N}, \boldsymbol{G}_{T}$ and $\boldsymbol{G}_{U}$ are wrench matrices associated with $\boldsymbol{f}_{N}, \boldsymbol{f}_{T}$ and $\boldsymbol{f}_{U}$, respectively.

The derivation of the motion equation of the rigid hand parallels that of Equation(1). Let $n_{\theta}$ be the number of joints in the hand, and $\tau$ be the $n_{\theta}$-vector of applied torques for

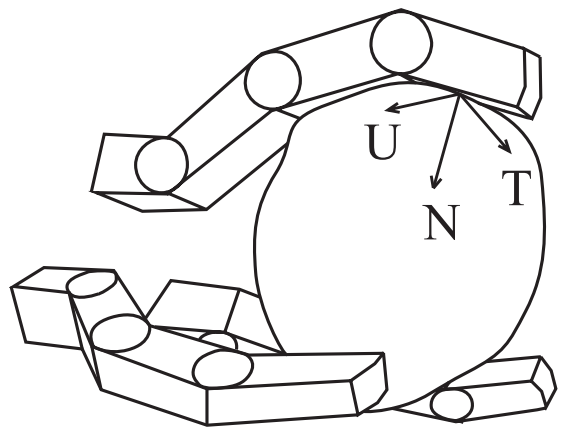

Figure 1: General whole arm grasp systems.

a revolute joint, respectively. The motion equation of the hand can be written as

$$
\boldsymbol{M}_{a} \ddot{\boldsymbol{\theta}}=\boldsymbol{\tau}-\boldsymbol{J}_{N}^{T} \boldsymbol{f}_{N}-\boldsymbol{J}_{T}^{T} \boldsymbol{f}_{T}-\boldsymbol{J}_{U}^{T} \boldsymbol{f}_{U}-\boldsymbol{g}_{a}
$$

where $\ddot{\boldsymbol{\theta}}$ is the $n_{\theta}$-vector of joint accelerations. $\boldsymbol{J}_{N}^{T}, \boldsymbol{J}_{T}^{T}$ and $\boldsymbol{J}_{U}^{T}$ are the hand Jacobian matrices associated with $\boldsymbol{f}_{N}, \boldsymbol{f}_{T}$ and $\boldsymbol{f}_{U}$, respectively. $\boldsymbol{M}_{a}$ is the $n_{\theta} \times n_{\theta}$-inertia matrix of the hand. $\boldsymbol{g}_{a}$ is the vectors of joint torques caused by external wrenches and velocity product wrenches.

According to Coulomb's law, the contact forces at rolling contacts lie within the boundary of its corresponding friction cone. The constraint on the $i$ th contact forces can be written as nonlinear inequalities

$$
\sqrt{f_{T R i}^{2}+f_{U R i}^{2}} \leq \mu_{i} f_{N R i}
$$

where $\mu_{i}$ is the coefficient of friction. In order to linearize Equation(3), the friction cone is approximated by the friction pyramid circumscribing the friction cone as shown in Figure 2. The friction pyramid can be written as

$$
\begin{aligned}
-\mu_{i} f_{N R i} & \leq f_{T R i} \leq \mu_{i} f_{N R i} \\
-\mu_{i} f_{N R i} & \leq f_{U R i} \leq \mu_{i} f_{N R i}
\end{aligned}
$$

Since the contact forces at both rolling and sliding contacts must be non-tensile, the normal contact forces satisfy

$$
f_{N R i} \geq 0, \quad f_{N S i} \geq 0
$$

Summing Equations(4),(5),(6) for all contacts yields

$$
\boldsymbol{B}_{\mu} \boldsymbol{f}_{A} \geq \mathbf{0}
$$

where

$$
\begin{aligned}
\boldsymbol{f}_{A}^{T} & =\left[\boldsymbol{f}_{N S}^{T}, \boldsymbol{f}_{N R}^{T}, \boldsymbol{f}_{T R}^{T}, \boldsymbol{f}_{U R}^{T}\right] \in \Re^{3 n_{R}+n_{S}} \\
\boldsymbol{B}_{\mu} & =\left[\begin{array}{ccc}
\boldsymbol{E}_{S} & \mathbf{0} \\
\mathbf{0} & \boldsymbol{U}_{R} & \boldsymbol{E}_{R} \\
\boldsymbol{U}_{R} & -\boldsymbol{E}_{R}
\end{array}\right] \\
\boldsymbol{U}_{R} & =\operatorname{diag}\left[\cdots,-\mu_{i}, \cdots\right] \in \Re^{n_{R} \times n_{R}}
\end{aligned}
$$




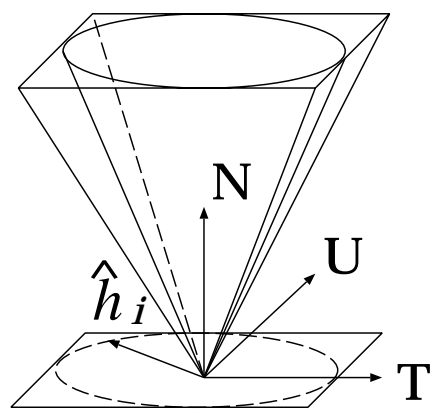

Figure 2: Friction cone and friction pyramid model.

$\boldsymbol{E}_{S}$ and $\boldsymbol{E}_{R}$ are $n_{S} \times n_{S}$ and $n_{R} \times n_{R}$ unit matrices, respectively. $\mathbf{0}$ is zero vector or matrix with appropriate dimension.

On the other hand, the contact forces at sliding contacts lie on the boundary of the corresponding friction cone and its tangential forces act on the object in the opposite direction to the motion of object relative to the hand at the contact point. The direction of relative motion is given by

$$
\widehat{u}_{i}=\frac{1}{\sqrt{\left(v_{T S i}\right)^{2}+\left(v_{U S i}\right)^{2}}}\left[\begin{array}{l}
v_{T S i} \\
v_{U S i}
\end{array}\right]
$$

where $v_{T S i}$ and $v_{U S i} \in \Re$ are relative sliding velocities in the direction of $T$ and $U$ with respect to the $i$ th contact frame, respectively. Therefore the tangential contact forces in the $i$ th contact frame are given by

$$
f_{T S i}=U_{T S i} f_{N S i}, \quad f_{U S i}=U_{U S i} f_{N S i}
$$

where

$$
U_{\bullet S i}=\frac{-\mu_{i} v_{\bullet S i}}{\sqrt{\left(v_{T S i}\right)^{2}+\left(v_{U S i}\right)^{2}}}, \quad \bullet \in\{T, U\}
$$

Summing Equation(9) for all sliding contacts yields

$$
\boldsymbol{f}_{T S}=\boldsymbol{U}_{T S} \boldsymbol{f}_{N S} \in \Re^{n_{S}}, \quad \boldsymbol{f}_{U S}=\boldsymbol{U}_{U S} \boldsymbol{f}_{N S} \in \Re^{n_{S}}
$$

where

$$
\boldsymbol{U}_{\bullet S}=\operatorname{diag}\left[\cdots, U_{\bullet S i}, \cdots\right] \in \Re^{n_{s} \times n_{s}}, \quad \bullet \in\{T, U\}
$$

Substituting Equation(11) into Equations(1),(2), the motion equations and friction constraints can be rewritten for all contacts as

$$
\begin{aligned}
\boldsymbol{M}_{o} \ddot{\boldsymbol{q}} & =\boldsymbol{G}_{A \mu} \boldsymbol{f}_{A}+\boldsymbol{g}_{o} \\
\boldsymbol{M}_{a} \ddot{\boldsymbol{\theta}} & =\boldsymbol{\tau}-\boldsymbol{J}_{A \mu}^{T} \boldsymbol{f}_{A}-\boldsymbol{g}_{a} \\
\boldsymbol{B}_{\mu} \boldsymbol{f}_{A} & >\mathbf{0}
\end{aligned}
$$

where

$$
\begin{gathered}
\boldsymbol{G}_{A \mu}=\left[\boldsymbol{G}_{N S}+\boldsymbol{G}_{T S} \boldsymbol{U}_{T S}+\boldsymbol{G}_{U S} \boldsymbol{U}_{U S}, \boldsymbol{G}_{N R}, \boldsymbol{G}_{T R}, \boldsymbol{G}_{U R}\right] \\
\in \Re^{6 \times\left(3 n_{R}+n_{S}\right)} \\
\boldsymbol{J}_{A \mu}^{T}=\left[\boldsymbol{J}_{N S}^{T}+\boldsymbol{J}_{T S}^{T} \boldsymbol{U}_{T S}+\boldsymbol{J}_{U S}^{T} \boldsymbol{U}_{U S}, \boldsymbol{J}_{N R}^{T}, \boldsymbol{J}_{T R}^{T}, \boldsymbol{J}_{U R}^{T}\right] \\
\in \Re^{n_{\theta} \times\left(3 n_{R}+n_{S}\right)}
\end{gathered}
$$

$\boldsymbol{G}_{N S}, \boldsymbol{G}_{T S}$ and $\boldsymbol{G}_{U S}$ are $6 \times n_{S}$ wrench matrices in the normal and tangential direction for sliding contacts. $\boldsymbol{G}_{N R}, \boldsymbol{G}_{T R}$ and $\boldsymbol{G}_{U R}$ are $6 \times n_{R}$ wrench matrices in the normal and tangential direction for rolling contacts. $\boldsymbol{J}_{N S}, \boldsymbol{J}_{T S}, \boldsymbol{J}_{N R}, \boldsymbol{J}_{T R}$ and $\boldsymbol{J}_{U R}$ are defined similarly.

\subsection{Kinematic model}

The $i$ th contact velocity on the object surface with respect to the $i$ th contact frame is given by $\left[\boldsymbol{G}_{N i} \boldsymbol{G}_{T i} \boldsymbol{G}_{U i}\right]^{T} \dot{\boldsymbol{q}}$. Similarly, the $i$ th contact velocity on the link surface is given by $\left[\begin{array}{lll}\boldsymbol{J}_{N i}^{T} & \boldsymbol{J}_{T i}^{T} & \left.\boldsymbol{J}_{U i}^{T}\right]^{T} \dot{\boldsymbol{\theta}} \text {. Therefore, }\end{array}\right.$ the $i$ th relative velocity, $v_{N i}, v_{T i}, v_{U i} \in \Re$, in the $N, T$, and $U$ direction with respect to the $i$ th contact frame can be written as

$$
\left[\begin{array}{c}
\boldsymbol{G}_{N i}^{T} \\
\boldsymbol{G}_{T i}^{T} \\
\boldsymbol{G}_{U i}^{T}
\end{array}\right] \dot{\boldsymbol{q}}-\left[\begin{array}{c}
\boldsymbol{J}_{N i} \\
\boldsymbol{J}_{T i} \\
\boldsymbol{J}_{U i}
\end{array}\right] \dot{\boldsymbol{\theta}}=\left[\begin{array}{c}
v_{N i} \\
v_{T i} \\
v_{U i}
\end{array}\right]
$$

As long as the contacts between the hand and the object are maintained, the normal relative velocities for both rolling and sliding contacts become

$$
v_{N R i}=0, \quad v_{N S i}=0
$$

In addition to Equation(16), the tangential relative velocities at the rolling contact must satisfy

$$
v_{T R i}=0, \quad v_{U R i}=0
$$

Imposing Equations(16), (17) on Equation(15), and summing it for all contacts yields

$$
\begin{aligned}
& \boldsymbol{G}_{A}^{T} \dot{\boldsymbol{q}}-\boldsymbol{J}_{A} \dot{\boldsymbol{\theta}}=\mathbf{0} \\
& \boldsymbol{G}_{\bullet S}^{T} \dot{\boldsymbol{q}}-\boldsymbol{J}_{\bullet S} \dot{\boldsymbol{\theta}}=\boldsymbol{v}_{\bullet S}, \quad \bullet \in\{T, U\}
\end{aligned}
$$

where

$$
\begin{aligned}
\boldsymbol{G}_{A} & =\left[\boldsymbol{G}_{N S}, \boldsymbol{G}_{N R}, \boldsymbol{G}_{T R}, \boldsymbol{G}_{U R}\right] \in \Re^{6 \times\left(3 n_{R}+n_{S}\right)} \\
\boldsymbol{J}_{A}^{T} & =\left[\boldsymbol{J}_{N S}^{T}, \boldsymbol{J}_{N R}^{T}, \boldsymbol{J}_{T R}^{T}, \boldsymbol{J}_{U R}^{T}\right] \in \Re^{n_{\theta} \times\left(3 n_{R}+n_{S}\right)}
\end{aligned}
$$

\section{Properties of manipulation system}

We cannot always plan arbitrary manipulation for a given manipulation system. Feasible manipulation is restricted by the property of kinematics and dynamics of the manipulation system. In this section, we show four properties of manipulation system based on kinematics and dynamics, and derive conditions which should be considered in motion planning.

The motion of object and hand is constrained so as to satisfy kinematics given by Equation(18). The issues which should be considered in motion planning concerning manipulation kinematics are whether, for a current contact mode, (i)there exists feasible joint velocity, $\dot{\boldsymbol{\theta}}$, for a desired object velocity, $\dot{\boldsymbol{q}}$, and (ii)the object velocity, $\dot{\boldsymbol{q}}$, is uniquely determined for a given joint velocity, $\dot{\boldsymbol{\theta}}$. These two problems depend on the properties of matrices $G_{A}$ and $\boldsymbol{J}_{A}[6],[12]$. 
Definition 1 A manipulation system is said to be kinematically manipulable if the hand Jacobian, $\boldsymbol{J}_{A} \in$ $\Re^{\left(3 n_{R}+n_{S}\right) \times n_{\theta}}$, is full rank and $3 n_{R}+n_{S} \leq n_{\theta}$.

If a manipulation system is kinematically manipulable for a current contact mode, we can find a joint velocity, $\dot{\boldsymbol{\theta}}$, which can accommodate an arbitrary object velocity, $\dot{\boldsymbol{q}}$.

Definition 2 A manipulation system is said to be kinematically determinate if the wrench matrix, $\boldsymbol{G}_{A} \in$ $\Re^{6 \times\left(3 n_{R}+n_{S}\right)}$, is full rank and $3 n_{R}+n_{S} \geq 6$.

When a manipulation system is kinematically determinate, the object velocity, $\dot{\boldsymbol{q}}$, is uniquely determined for a given joint velocity, $\dot{\boldsymbol{\theta}}$. Otherwise there are infinite solutions for Equation(18), and the object cannot be manipulated by the joint motion.

On the other hand, manipulation dynamics is determined by Equations $(12) \sim(14)$ and acceleration kinematics obtained by differentiating Equation(18)

$$
\boldsymbol{G}_{A}^{T} \ddot{\boldsymbol{q}}-\boldsymbol{J}_{A} \ddot{\boldsymbol{\theta}}+\dot{\boldsymbol{G}}_{A}^{T} \dot{\boldsymbol{q}}-\dot{\boldsymbol{J}}_{A} \dot{\boldsymbol{\theta}}=\mathbf{0}
$$

The issues which should be considered in motion planning concerning manipulation dynamics are whether (i)there exists feasible joint driving torque, $\tau$, to generate a desired object acceleration, $\ddot{\boldsymbol{q}}$, and (ii)we can uniquely predict an object acceleration, $\ddot{\boldsymbol{q}}$, for a given joint driving torque, $\boldsymbol{\tau}$.

Definition 3 A manipulation system satisfying the following conditions (i),(ii) and (iii) simultaneously is said to be dynamically manipulable. (i)a manipulation system is kinematically manipulable, (ii) $\boldsymbol{G}_{A \mu} \in \Re^{6 \times\left(3 n_{R}+n_{S}\right)}$ is full rank and $3 n_{R}+n_{S} \geq 6$ and (iii) $\boldsymbol{J}_{A \mu} \in \Re^{\left(3 n_{R}+n_{S}\right) \times n_{\theta}}$ is full rank.

When the condition(i) holds, the hand Jacobian $\boldsymbol{J}_{A}$ becomes row full rank and there exists feasible joint acceleration, $\ddot{\boldsymbol{\theta}}$, satisfying Equation(20) for an arbitrary $\ddot{\boldsymbol{q}}$. In order to exist contact force, $\boldsymbol{f}_{A}$, satisfying Equations(12),(14) for an arbitrary $\ddot{\boldsymbol{q}}$, the manipulation system should satisfy the condition(ii). In addition, if condition(iii) holds, joint torque, $\boldsymbol{J}_{A \mu}^{T} \boldsymbol{f}_{A}$, which can balance with any contact force, $\boldsymbol{f}_{A}$, can be generated. Since a joint driving torque, $\tau$, can be obtained by substituting $\ddot{\boldsymbol{\theta}}$ and $\boldsymbol{f}_{A}$ into Equation(13), we can get an unique $\boldsymbol{\tau}$ which generate $\ddot{\boldsymbol{q}}$ if the conditions(i),(ii) and (iii) hold simultaneously. Therefore it is necessary that a manipulation system should be dynamically manipulable so that we could find a joint driving torque generating a desired arbitrary object acceleration in motion planning.

Contacts between rigid bodies generate complementarity constraints on relative accelerations and the corresponding contact forces[11]. The problems on the existence of unique object acceleration for a given joint torque can be discussed by reducing the problems into a linear complementarity problem(LCP) which can be written as[14]

$$
\boldsymbol{f}_{A} \geq \mathbf{0}, \boldsymbol{a}=\boldsymbol{A} \boldsymbol{f}_{A}+\boldsymbol{b} \geq \mathbf{0}, \boldsymbol{f}_{A}^{T} \boldsymbol{a}=\mathbf{0}
$$

where

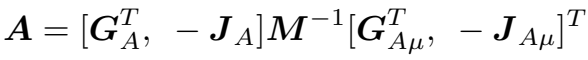

$$
\begin{aligned}
& \boldsymbol{b}=\left[\boldsymbol{G}_{A}^{T}, \quad-\boldsymbol{J}_{A}\right] \boldsymbol{M}^{-1}\left[\boldsymbol{g}_{o}^{T},\left(\boldsymbol{\tau}-\boldsymbol{g}_{a}\right)^{T}\right]^{T} \\
& +\left[\dot{\boldsymbol{G}}_{A}^{T},-\dot{\boldsymbol{J}}_{A}\right]\left[\dot{\boldsymbol{q}}^{T}, \dot{\boldsymbol{\theta}}^{T}\right]^{T} \\
& \boldsymbol{a}=\left[\boldsymbol{a}_{N S}^{T}, \boldsymbol{a}_{N R}^{T}, \boldsymbol{a}_{T R}^{T}, \boldsymbol{a}_{U R}^{T}\right]^{T} \\
& \boldsymbol{M}=\operatorname{diag}\left[\boldsymbol{M}_{o}, \boldsymbol{M}_{a}\right]
\end{aligned}
$$

$\boldsymbol{a} \in \Re^{3 n_{R}+n_{S}}$ is a relative acceleration with respect to a contact frame. The LCP has unique solutions $\boldsymbol{a}$ and $\boldsymbol{f}_{A}$ for all $\boldsymbol{b}$ if and only if the matrix $\boldsymbol{A}$ is a P-matrix[5]. Using this theory, we make a definition for uniqueness of dynamics as follows.

Definition 4 A manipulation system is said to be dynamically determinate if the matrix $\boldsymbol{A}$ is a P-matrix.

We notice that the vector $\boldsymbol{b}$ is a function of $\boldsymbol{\tau}$. If a manipulation system is dynamically determinate, contact forces, $\boldsymbol{f}_{A}$, can be uniquely determined for an arbitrary joint torque, $\tau$. In addition an object acceleration, $\ddot{\boldsymbol{q}}$, can be uniquely determined for the given $\boldsymbol{f}_{A}$ using Equation(12). In other words, it is found that such a manipulation system does not become indeterminate or inconsistent for a given joint torque.

We classified the properties of manipulation system based on kinematics and dynamics. Among these properties, the "kinematically determinate" and "dynamically determinate" manipulation systems guarantee that manipulation along with the desired object trajectory can be realized by applying joint velocity and joint torque which are obtained in motion planning, respectively. Therefore the validity of solutions for forward problem can be guaranteed. On the other hand, the "kinematically manipulable" and "dynamically manipulable" manipulation systems guarantee that there exist feasible joint velocity and joint torque to accommodate the arbitrary object motion. Therefore the validity of solutions for inverse problem can be guaranteed. In this paper, by changing contact modes, we plan the motions of the manipulation system to guarantee the valid solution for both forward and inverse problems. We can summarize the conditions on kinematics and dynamics mentioned above and the conditions on the contact number shown in the section 2.1 by the following equations. These conditions are used to decide the feasibility of manipulation in the motion planning next.

$$
\begin{array}{r}
n_{c}=n_{R}+n_{S} \leq n_{\theta} \\
6 \leq 3 n_{R}+n_{S} \leq n_{\theta} \\
\operatorname{rank}\left(\boldsymbol{J}_{A}\right)=3 n_{R}+n_{S}
\end{array}
$$




$$
\begin{array}{r}
\operatorname{rank}\left(\boldsymbol{J}_{A \mu}\right)=3 n_{R}+n_{S} \\
\operatorname{rank}\left(\boldsymbol{G}_{A}\right)=6 \\
\operatorname{rank}\left(\boldsymbol{G}_{A \mu}\right)=6
\end{array}
$$

$\boldsymbol{A} \in \mathrm{P}-$ matrix class

\section{Motion planning}

\subsection{Hybrid system}

We should notice that manipulation dynamics changes according to the change of contact modes. The manipulation system with switching contact modes consists of multiple subsystems corresponding to each contact mode as shown in Figure 3. The transition between subsystems occurs by switching a contact mode, and the dynamics and kinematics change accordingly. In other words, since the motion of such a manipulation system can be represented by a sequence of contact mode which can be expressed as discrete events as well as time continuous motion, we can regard the system as a hybrid system.

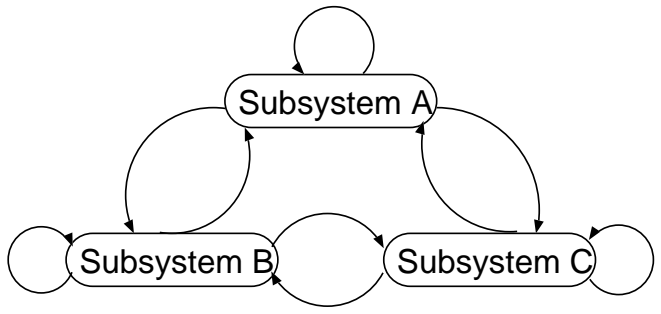

Figure 3: Transition between subsystems.

In order to plan the manipulation based on switching contact modes, we consider a state $\times$ time space of the manipulation system corresponding to the contact mode whenever that is switched, as shown in Figure 4. Let the state be the positions and velocities of the manipulation system. The manipulation time, $\left[0, t_{\text {goal }}\right]$, is divided into $t_{j}: 0<$ $t_{1}<\cdots<t_{N+1}=t_{\text {goal }}$ by the number of switches, $N$. Let $m$ be the dimension of the manipulation system. The state, $\boldsymbol{x}_{j} \in \mathcal{X}_{j} \subset \Re^{m}$, of the manipulation system on $\left[t_{j}, t_{j+1}\right]$ is governed by dynamics and kinematics for the state space, $\mathcal{X}_{j}$, of the manipulation system, corresponding to a contact mode, $\mathcal{M}_{j}$. The state trajectories which connects states $\boldsymbol{x}_{j}\left(t_{j}\right)$ with $\boldsymbol{x}_{j}\left(t_{j+1}\right)$ on $\left[t_{j}, t_{j+1}\right], j=0, \ldots, N$ and the corresponding joint torque trajectories are determined so as to satisfy Equations(22) (28). At a switching time, $t_{j+1}$, the state space changes from $\mathcal{X}_{j}$ to $\mathcal{X}_{j+1}$ and the state changes from $\boldsymbol{x}_{j}\left(t_{j+1}\right)$ to $\boldsymbol{x}_{j+1}\left(t_{j+1}\right)$ continuously.

We should decide the following three components in motion planning for the manipulation system with the property mentioned above.

(i) The sequence of contact modes, $\left\{\mathcal{M}_{j}\right\}_{j=0}^{N}$.

(ii) The switching times, $\left\{t_{j}\right\}_{j=1}^{N}$.

(iii) The state trajectories of manipulation system and the joint torque trajectories on each interval $\left[t_{j}, t_{j+1}\right]$.

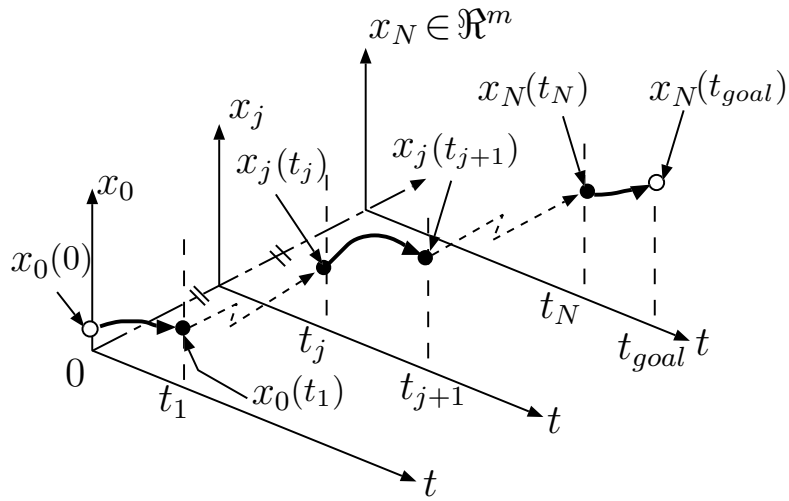

Figure 4: A sequence of state $\times$ time space of manipulation system corresponding to contact modes.

Since it is quite a difficult task to find all three components of optimal solution, we can find an approximate solution by defining the motion planning problem as; "Given the switching times and each object state trajectories, $\boldsymbol{q}(t), \dot{\boldsymbol{q}}(t), t \in\left[t_{j}, t_{j+1}\right], j=0, \ldots, N$, find feasible joint trajectories of motion and driving torques which connect an object initial state, $(\boldsymbol{q}(0), \dot{\boldsymbol{q}}(0))$, with an object goal state, $\left(\boldsymbol{q}\left(t_{\text {goal }}\right), \dot{\boldsymbol{q}}\left(t_{\text {goal }}\right)\right)$, utilizing rolling and/or sliding contacts." Note that the object trajectory is assumed to be specified in motion planning based on an inverse problem. However this trajectory is modified iteratively so that the object can be actually manipulated to the goal. The algorithm for the three components are organized in the flow chart in Figure 5. We will next describe each of these components and the organization.

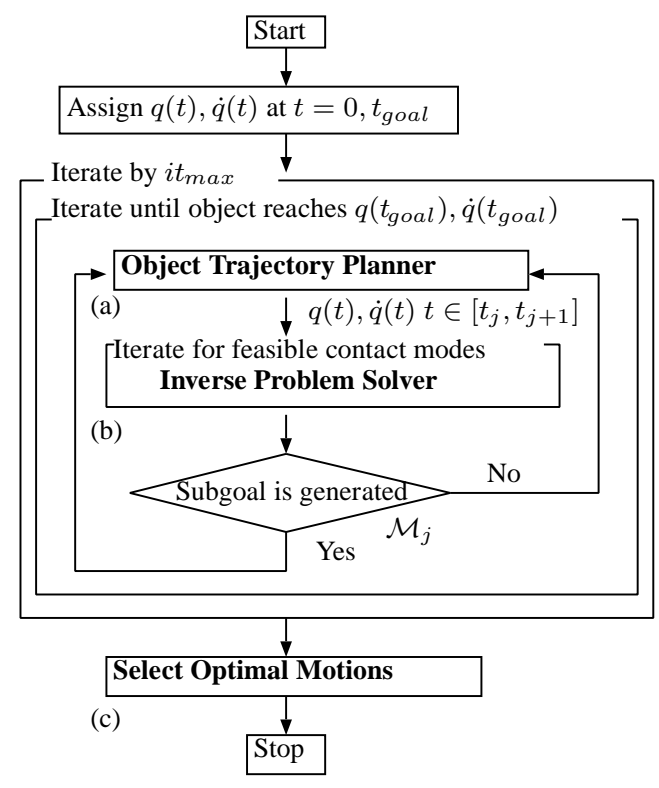

Figure 5: An algorithm of motion planning.

\subsection{Algorithm}

The proposed motion planning, as shown in Figure 5, consists of (a) a planning of object state trajectory, $\boldsymbol{q}(t)$ 
and $\dot{\boldsymbol{q}}(t)$, on $\left[t_{j}, t_{j+1}\right]$, (b) an execution of the inverse problem for the given object state trajectory and a generation of subgoals (the contact modes which make manipulation feasible on $\left[t_{j}, t_{j+1}\right]$ and the states of manipulation system at the switching time, $t_{j+1}$ ), and (c) a selection of desirable trajectories. The (a) and (b) are executed iteratively whenever the assignment of switching time, and the subgoals at each switching time are generated consequently. Subgoals which connects an object initial state with an object goal state are finally obtained, and the transition of manipulation system are expressed by contact mode transition graphs. We will next discuss (a),(b) and (c) in detail.

At a switching time, $t_{j}$, a next switching time, $t_{j+1}$, is assigned and the object state trajectory, $\boldsymbol{q}(t), \dot{\boldsymbol{q}}(t)$, on $\left[t_{j}, t_{j+1}\right]$ are planned in (a). Since an object state $\times$ time space is generally high-dimensions, we try to reduce the dimension of search space by utilizing an object desirable rough position trajectory, $\widetilde{\boldsymbol{q}}(t), t \in\left[0, t_{\text {goal }}\right]$, which connects an object initial position, $\boldsymbol{q}(0)$, with an object goal position, $\boldsymbol{q}\left(t_{\text {goal }}\right)$. The rough object position trajectory is called a nominal trajectory. Now as shown in Figure 6, the next switching time, $t_{j+1}$, is assigned randomly along with a time axis for a current switching time, $t_{j}$, and the object position, $\widetilde{\boldsymbol{q}}\left(t_{j+1}\right)$ on the nominal trajectory is used as boundary values of the object trajectory, $\boldsymbol{q}(t)$. Therefore boundary conditions for $\boldsymbol{q}(t)$ can be written as

$$
\boldsymbol{q}\left(t_{j}\right)=\widetilde{\boldsymbol{q}}\left(t_{j}\right), \quad \boldsymbol{q}\left(t_{j+1}\right)=\widetilde{\boldsymbol{q}}\left(t_{j+1}\right)
$$

A relative tangential velocity at each contact point should be zero at each switching time so that we can assign any contact mode at the switching time. That can be realized when the object velocity at each switching time is given so that

$$
\dot{\boldsymbol{q}}\left(t_{j}\right)=\dot{\boldsymbol{q}}\left(t_{j+1}\right)=0
$$

The object state trajectory, $\boldsymbol{q}(t), \dot{\boldsymbol{q}}(t)$, on $\left[t_{j}, t_{j+1}\right]$, are generated so as to satisfy Equations(29),(30). The idea of generating an object state trajectory by giving a switching time randomly, rather that directly sampling the state space of the object, make the exploration quickly over the state $x$ time space and the planner find feasible solutions easily.

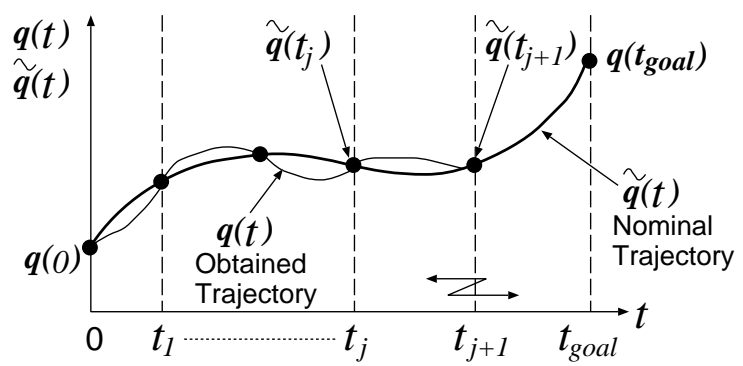

Figure 6: Generating object trajectory on $\left[t_{j}, t_{j+1}\right]$ based on randomized approach for nominal trajectory.

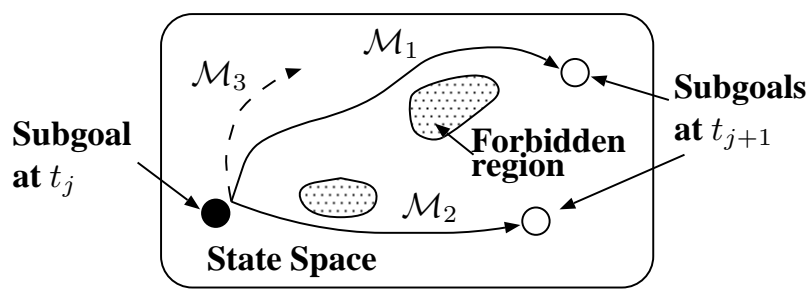

Figure 7: Building subgoals at switching time, $t_{j+1}$, by exploring the state space of the manipulation system corresponding to contact modes $\mathcal{M}_{1}, \mathcal{M}_{2}$ and $\mathcal{M}_{3}$. Forbidden regions where there does not exist solution are detected by the execution of inverse problem.

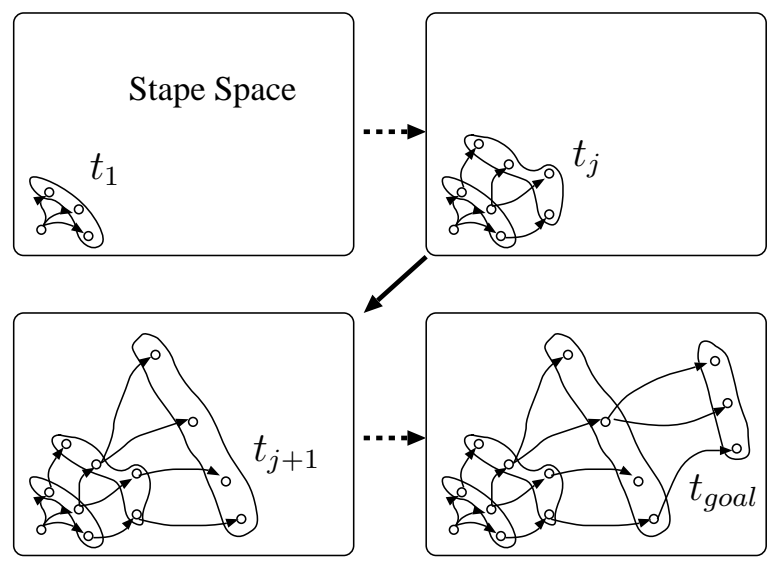

Figure 8: Iterative process of subgoal expansion on $\left[0, t_{\text {goal }}\right]$.

Figure 7 shows the process of exploration of the state space of manipulation system on intervals, $\left[t_{j}, t_{j+1}\right]$, in (b). Black and white circles show the subgoals at the switching time $t_{j}$ and $t_{j+1}$, respectively. A line with arrow indicates the state trajectory of the manipulation system. Whenever a new switching time, $t_{j+1}$, is assigned, the subsystems corresponding to the combinations of contact modes satisfying Equations(22),(23) are considered, and the inverse problem is executed iteratively at ever instant on $\left[t_{j}, t_{j+1}\right]$ for the assigned object state trajectory. When solutions satisfying Equations(22) (28) over $\left[t_{j}, t_{j+1}\right]$ are obtained, the subgoals at time $t_{j+1}$ are generated. If subgoals cannot be generated for any contact mode, a new object trajectory is given and the step (b) is executed iteratively. Note that there may exist multiple subgoals at the same switching time, $t_{j+1}$, as shown in Figure 7, because system dynamics is different according to a given contact mode. Figure 8 shows the iterative process of system state exploration on $\left[0, t_{\text {goal }}\right]$ using this algorithm.

Iterating a generation of subgoals reaching the object goal state up to $i t_{\max }$, the contact mode transition which minimizes a performance index of the form

$$
f_{\text {cost }}=\sum_{j=0}^{N} \int_{t_{j}}^{t_{j+1}} P d t
$$


is selected in (c). A cost function, $P$, about energy, joint torque and so on is considered according to the aim of manipulation.

\subsection{Derivation of solutions for inverse problem}

In this section we now derive solutions for inverse problem which is defined as "Given object motions, $\ddot{\boldsymbol{q}}(t), \dot{\boldsymbol{q}}(t)$ and $\boldsymbol{q}(t)$, at an instant and a contact mode at each contact point, find joint driving torque, $\boldsymbol{\tau}(t)$, and the state of the manipulation system at a next instant". When the manipulation system is dynamically manipulable for a current contact mode, there exists a feasible $\boldsymbol{\tau}(t)$ for a given $\ddot{\boldsymbol{q}}(t)$. However we cannot always decide unique $\tau(t)$ due to the following two reasons.

When the wrench matrix, $\boldsymbol{G}_{A \mu}$, is over-constrained, that is, $\boldsymbol{G}_{A \mu}$ is full rank and $3 n_{R}+n_{S}>6$, there are multiple solutions to the contact force for a given object acceleration. We determine the minimum norm of the contact force, $\boldsymbol{f}_{A}$, by solving the following quadratic programming problem,

$$
\begin{aligned}
\text { minimize } & 1 / 2 \boldsymbol{f}_{A}^{T} \boldsymbol{f}_{A} \\
\text { subject to } & \text { Eqs.(12), (14) }
\end{aligned}
$$

On the other hand, when the manipulation system is kinematically manipulable for a current contact mode and the hand has redundant degrees of freedom(dof) for a admissible dof of contact mode, that is, $\boldsymbol{J}_{A}$ is full rank and $n_{\theta}>3 n_{R}+n_{S}$, there are multiple solutions to the joint acceleration for a given object acceleration. We derive the joint acceleration, $\ddot{\boldsymbol{\theta}}$, from Equation(20), using the generalized inverse of $\boldsymbol{J}_{A}$, as

$$
\ddot{\boldsymbol{\theta}}=\boldsymbol{J}_{A}^{+}\left(\boldsymbol{G}_{A}^{T} \ddot{\boldsymbol{q}}+\dot{\boldsymbol{G}}_{A}^{T} \dot{\boldsymbol{q}}-\dot{\boldsymbol{J}}_{A} \dot{\boldsymbol{\theta}}\right)
$$

Given object motions, $\ddot{\boldsymbol{q}}(t), \dot{\boldsymbol{q}}(t)$ and $\boldsymbol{q}(t)$, and joint states, $\boldsymbol{\theta}(t)$ and $\dot{\boldsymbol{\theta}}(t)$, the joint acceleration, $\ddot{\boldsymbol{\theta}}(t)$, can be obtained from Equation(33). Substituting $\dot{\boldsymbol{q}}(t)$ and $\dot{\boldsymbol{\theta}}(t)$ into Equation(19) yields the sliding velocity, $\boldsymbol{v}_{\bullet S}(t), \bullet \in$ $\{T, U\}$. Substituting $\ddot{\boldsymbol{q}}(t)$ and $\boldsymbol{v}_{\bullet}(t)$ into Equation(32) yields $\boldsymbol{f}_{A}(t)$ which should be applied to the object in order to generate $\ddot{\boldsymbol{q}}(t)$. Therefore the joint driving torque, $\boldsymbol{\tau}(t)$, to generate a desired object motions is derived by substituting $\ddot{\boldsymbol{\theta}}(t)$ and $\boldsymbol{f}_{A}(t)$ into Equation(13). The joint state, $\boldsymbol{\theta}(t+\Delta t)$ and $\dot{\boldsymbol{\theta}}(t+\Delta t)$, at a next instant is obtained by integration of $\ddot{\boldsymbol{\theta}}(t)$.

\section{Simulation results}

For simplicity, we now consider planning a 2D whole arm grasp system in order to lift up an object toward a palm. As shown in Figure 9, a two 2-DOF fingered hand with a fixed palm is used to manipulate an elliptical object in the vertical plane with rolling and/or sliding contacts. Each 2 nd link of the both fingers has the contacts with the object at the initial time. We assume that the contacts are not broken once the links have the contact with the object. A base frame is placed in the midpoint between each first joint in the palm.

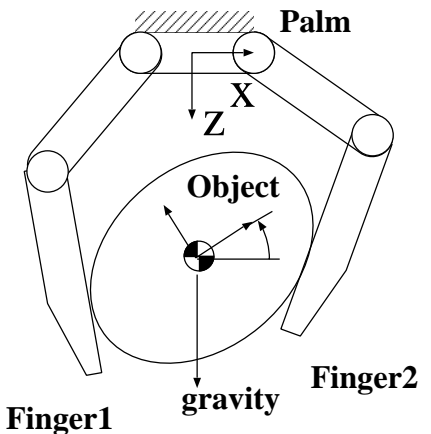

Figure 9: A planar whole arm grasp.

In the case of manipulation by hand with four joints in a plane, the following constraints about the number of rolling and sliding contacts are imposed by Equations(22),(23):

$$
n_{R}+n_{S} \leq 4, \quad 3 \leq 2 n_{R}+n_{S} \leq 4
$$

The feasible contact mode set satisfying Equation(34) is

$$
\mathcal{M} \subseteq\{R R, R S, 2 S R, 3 S, 4 S\}
$$

The inverse problem is executed for a manipulation system corresponding to $\mathcal{M}$, according to the number of a current contact point.

The manipulation task is to rotate the object from $20 \mathrm{de}-$ gree to 0 degree, and to translate it from $0.30 \mathrm{~m}$ to $0.15 \mathrm{~m}$ in the $z$ direction in two seconds. The object has a major axis of $0.37 \mathrm{~m}$ and its minor axis is $0.30 \mathrm{~m}$. The mass of the object is $3.00 \mathrm{~kg}$, and the moment of inertia about the center of mass is $4.25 \times 10^{-2} \mathrm{~kg} \cdot \mathrm{m}^{2}$. The fixed palm of the hand is $0.20 \mathrm{~m}$ long. The length of each finger link is $0.30 \mathrm{~m}$. The mass of the finger link is $0.5 \mathrm{~kg}$ with a moment of inertia of $1.67 \times 10^{-3} \mathrm{~kg} \cdot \mathrm{m}^{2}$. The coefficient of friction is $\mu=0.2$.

We obtained three contact mode transition graphs for $i t_{\max }=2$, as shown in Figure 10. Subscripts of contact mode show a number of hand getting in contact with the object. The figures on a edge of the graph indicate a sequence of switching. The contact mode transition with the minimum consumption of joint driving torques is $R_{1} R_{2} \rightarrow$ $R_{1} R_{2} \rightarrow S_{1} R_{2} \rightarrow R_{1} R_{2}$, as shown in Figure 10(a). The snap shots for this solution are shown in Figures 11. The 2nd links with rolling and sliding contacts are drawn by thick and thin lines, respectively.

\section{Concluding remarks}

We have presented the mathematical conditions and an algorithm for the whole arm grasp planning problem with switching contact modes. Changing the contact modes allows manipulation skill to be extended. Motion planning 


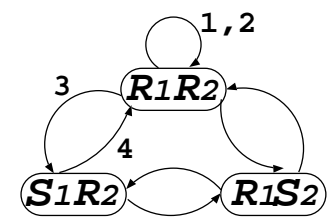

(a)

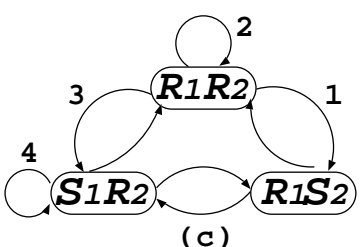

Figure 10: Contact mode transition graph.

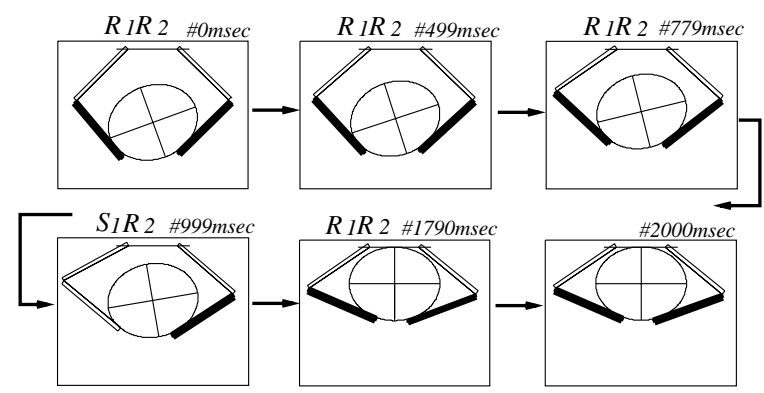

Figure 11: Snap shots of whole arm grasps.

for such a system should consider changing dynamics and kinematics according to the contact modes. We showed four properties of manipulation system based on dynamics and kinematics, and derived conditions to be satisfied in motion planning. The conditions restrict the feasible contact mode and the number of contact points.

The planning problem is simplified by giving the object trajectory using a randomized technique. Therefore it is not guaranteed to find a trajectory whenever one exists. Though the algorithm is not complete, we could find the solution trajectory efficiently compared to the direct sampling approach. Future works focus on generating a nominal object trajectory by a randomized approach although the proposed algorithm requires to specify an appropriate nominal trajectory a priori such that there exist solutions at each instant.

\section{Acknowledge}

It is a pleasure to thank Dr. Vijay Kumar, University of Pennsylvania, for helpful comments.

\section{References}

[1] N.M.Amato, Y.Wu. A randomized roadmap method for path and manipulation planning. In Proc. of IEEE Int. Conf. on Robotics and Automation, 1996.

[2] J.Barraquand, J.C.Latombe. Robot motion planning: A distributed representation approach. Int. Journal of Robotics Research, 10(6):628-649, 1991.

[3] M.S.Branicky, V.Borkar, S.Mitter. A unified framework for hybrid control. In Proc. of 33rd IEEE Conf. on Decision and Cotrol, pages 4228-44234, 1994.
[4] M.J.Cherif, K.K.Gupta. Planning quasi-static fingertip manipulations for reconfiguring objects. IEEE Transaction on Robotics and Automation, 15(5):837-848, 1999.

[5] R.W.Cottle, J.Pang, R.E.Stone. The Linear Complementarity Problem. Academic Press, 1992.

[6] L.Han, J.C.Trinkle. The instantaneous kinematics of manipulation. In Proc. of IEEE Int. Conf. on Robotics and Automation, 1998.

[7] L.Kavraki, J.C.Latombe. Randomized preprocessing of configuration space for fast path planning. In Proc. of IEEE Int. Conf. on Robotics and Automation, 1994.

[8] R.Kindel, D.Hsu, J.C.Latombe, S.Rock. Kinodynamic motion planning amidst moving obstacles. In Proc. of IEEE Int. Conf. on Robotics and Automation, 2000.

[9] S.M.LaValle, J.J.Kuffner,Jr. Randomized kinodynamic planning. In Proc. of IEEE Int. Conf. on Robotics and Automation, 1999.

[10] M.D.Lemmon, K.X.He, I.Markovsky. Supervisory hybrid systems. IEEE Control Systems, August:42-55, 1999.

[11] P.Lötstedt. Coulomb friction in two-dimensional rigid body systems, Zeitschrift Für Angewandte Mathematik und Mechanik, 61:605-615, 1981.

[12] D.J.Montana. The kinematics of multi-fingered manipulation. IEEE Transaction on Robotics and Automation, 11(4):491-503, 1995.

[13] J.C.Trinkle, J.J.Hunter. A framework for planning dextrous manipulation. In Proc. of IEEE Int. Conf. on Robotics and Automation, pages 1245-1251, 1991.

[14] J.C.Trinkle, J.S.Pang, S.Sudarsky, G.Lox. On dynamics multi-rigid-body contact problems with Coulomb friction. Zeitschrift Für Angewandte Mathematik und Mechanik, 77(4):267-279, 1997.

[15] M.Zefran, J.P.Desai, V.Kumar. Continuous motion plans for robotics systems with changing dynamic behavior. In Algorithmic Foundations of Robotics, 1996. 